\title{
EXPERIENCIA EN EL ANÁLISIS DE LAS ESTRATEGIAS DE AFRONTAMIENTO EN CÁNCER DE MAMA CON EL APOYO DEL PROGRAMA NVIVO
}

\author{
Elena Torralba-Martínez ${ }^{1}$ y Núria Codern-Bové ${ }^{2}$ \\ ${ }^{1}$ Universitat Autònoma de Barcelona, España. elenatm4@gmail.com \\ ${ }^{2}$ Escola Universitària d'Infermeria i Teràpia Ocupacional de Terrassa (EUIT), Universitat Autònoma de Barcelona, \\ Terrassa, España. nuriacodern@euit.fdsll.cat
}

\begin{abstract}
Resumen. Introducción: El cáncer de mama fue el más frecuente en España durante el año 2018. La angustia psicólogica, emocional y social de las mujeres es mediada por diferentes factores entre ellos el afrontamiento. Objetivos: Comprender la evolución de las estrategias de afrontamiento en las mujeres y su papel en la adaptación al cáncer de mama. Métodos: Estudio cualitativo interpretativo con enfoque fenomenológico. La muestra incluyó 13 mujeres de 50 a 70 años diagnosticadas de cáncer de mama durante 2016. Se utilizó entrevista semi-estructurada grabada en audio para la recogida de datos. Se realizó un análisis de contenido temático asistido por el programa Nvivo v.12. Resultados: En la fase aguda se aprecia heterogeneidad en el uso de las estrategias de afrontamiento, la aceptación de responsabilidad únicamente aparece en esta fase. En la fase extendida destacan las estrategias de búsqueda de apoyo social, distanciamiento y planificación. Por último, en la fase permanente, destacan estrategias de distanciamiento y se identifica la reevaluación positiva, estrategia presente únicamente en esta fase. Conclusiones. Las estrategias de afrontamiento evolucionan con el tiempo y permiten a la mujer adaptarse al cáncer de mama de forma específica, partiendo de la evaluación individual de la enfermedad como factor estresor.
\end{abstract}

Palabras clave: Cáncer de Mama; Afrontamiento; Supervivencia.

\section{EXPERIENCE IN THE ANALYSIS OF COPING STRATEGIES IN BREAST CANCER WITH THE SUPPORT OF THE NVIVO PROGRAM}

\begin{abstract}
Introduction: Breast cancer was the most frequent Spain during the year 2018. The psychological, emotional and social anguish of women is mediated by different factors including coping. Goals. To understand the evolution of coping strategies in women and its role in adaptation to breast cancer. Methods: Qualitative interpretive study with phenomenological approach. The sample included 13 women aged 50 to 70 diagnosed with breast cancer during 2016. A semistructured interview recorded in audio was used for data collection. An analysis of thematic content was carried out by the Nvivo v.12 program. Results: In the acute phase there is heterogeneity in the use of coping strategies, the acceptance of responsibility only appears in this phase. In the extended phase, strategies for seeking social support, distancing and planning stand out. Finally, in the permanent phase, distancing strategies stand out and positive reevaluation is identified, a strategy only present in this phase. Conclusions. Coping strategies evolve over time and allow women to adapt to breast cancer specifically, based on the individual assessment of the disease as a stressor.
\end{abstract}

Keywords: Breast Neoplasms; Coping; Survivorship.

\section{INTRODUCCIÓN}

Según la Agencia Internacional de Investigación sobre el cáncer, el más frecuente en España durante el año 2018 fue el cáncer de mama en las mujeres, con una incidencia estimada de 75,4 casos por cada 100.000 habitantes (GLOBOCAN, 2018). Durante la 
enfermedad, la angustia psicológica, emocional y social de enfrentarse a un proceso como el cáncer de mama es mediada por diferentes factores, entre ellos el afrontamiento (Cope, 1995; Kaur et al., 2008; Zhang et al., 2019). Este se puede entender como "esfuerzo por reducir o mitigar los efectos aversivos del estrés, los cuales pueden ser psicológicos o conductuales" (Everly, 1989, p. 44).

La teoría transaccional del estrés de Lazarus y Folkman (1984) mantiene que las evaluaciones mentales realizadas cuando se enfrenta una situación estresante, como el cáncer de mama, están relacionadas con los resultados de afrontamiento. La evaluación se considera un proceso mediante el cual las personas deciden si las situaciones estresantes son amenazas para su bienestar o no (evaluaciones primarias), y determinan si tienen los recursos para lidiar con el factor estresante cuando se considera una amenaza (evaluaciones secundarias) (Lazarus \& Folkman, 1984). Las mujeres evalúan el cáncer de mama como una situación amenazante que les provoca daños/pérdidas; pero también lo pueden llegar a evaluar como un desafío (Bigatti, Steiner, \& Miller, 2012; Palacios Espinosa, Lizarazo, Moreno, \& Ospino, 2015).

Durante los primeros meses tras el diagnóstico de cáncer de mama, las mujeres llegan a ser conscientes de la amenaza de muerte que supone la enfermedad, pero al mismo tiempo se sienten esperanzadas y optimistas, y en general se esfuerzan para controlar las reacciones emocionales (do Carmo Pinto Coelho Paiva, de Oliveira Salimena, de Oliveira Souza, \& de Melo, 2015; Drageset, Lindstrøm, \& Underlid, 2010). En el periodo del tratamiento, tienden a utilizar un afrontamiento activo, centrado en el problema (Cha, Yoo, \& Cho, 2012). Y por último, en una etapa posterior al tratamiento activo, lo que equivaldría a la etapa aguda de supervivencia, vemos como estrategias de búsqueda de apoyo social se relacionan con un adecuado ajuste psicosocial (Jang \& Kim, 2018).

Diferentes estudios han apreciado como determinadas estrategias de afrontamiento (EA) se vinculan a mejor calidad de vida y resultan más adaptativas, mientras que otras estrategias se relacionan con un peor ajuste psicosocial y síntomas depresivos mayores (Edward, Chipman, Giandinoto, \& Robinson, 2019; Paek et al., 2016). Según la teoría transaccional el afrontamiento no es estático, sino que cambia con el tiempo (Lazarus \& Folkman, 1984). Por ello, nos planeamos responder la pregunta ¿Qué papel juegan las EA en la adaptación al cáncer de mama a lo largo del proceso de la enfermedad? El objetivo del presente estudio 
es comprender la evolución de las EA en las mujeres y su relación con la adaptación al cáncer de mama.

\section{METODOLOGÍA}

\subsection{Diseño}

Se llevó a cabo un estudio cualitativo interpretativo tomando la fenomenología como enfoque teórico-metodológico. Este enfoque ayudó a comprender el significado que las mujeres atribuyeron a su experiencia de cáncer de mama y su relación con las EA a través del tiempo.

\subsection{Selección de participantes y reclutamiento}

Se realizó un muestro intencional con representación teórica para seleccionar a las participantes con la intención de obtener variabilidad discursiva para responder a la pregunta de investigación. Se incluyeron mujeres diagnosticadas de cáncer de mama en el año 2016, con edades comprendidas entre los 50 y los 70 años, con diferentes ingresos económicos familiares, y con o sin tratamientos coadyuvantes. El tamaño de la muestra se determinó en base a la saturación de los datos. La identificación y búsqueda de las informantes se realizó a partir de la base de datos de un estudio previo realizado en un hospital de Barcelona durante 2016-2017. Una vez seleccionadas las mujeres, la enfermera de la Unidad de Mama del hospital contactó telefónicamente con ellas y les propuso participar en el estudio. Posteriormente, un miembro del equipo de investigación contactó telefónicamente con aquellas mujeres que habían aceptado y dado su consentimiento de forma telefónica. En esta segunda llamada se acordó un día para realizar la entrevista de forma presencial.

El estudio recibió la aprobación por parte del Comité de Ética de dicho hospital y las participantes firmaron el consentimiento informado previa participación en las entrevistas. Todos los procedimientos se realizaron de acuerdo con los estándares éticos de conformidad con la Ley Orgánica 15/1999 de España 13 de diciembre sobre la "Protección de datos personales" (Ley Orgánica 15/ 1999, 1999, p. 43088) y con la Declaración de Helsinki de 1964 y sus posteriores enmiendas o estándares éticos comparables (World Medical Association, 2008). 


\subsection{Recogida de información}

La recogida de información se realizó a través de una entrevista semi-estructurada teniendo en cuenta las diferentes esferas en las que se mueve la mujer (familiar, social y laboral) (Ferrell, Dow, \& Grant, 1995), y el proceso de la enfermedad desde el diagnóstico, posterior intervención y tratamiento, hasta la actualidad (trascurridos dos años desde el diagnóstico). Para probar la guía de la entrevista se realizó una entrevista piloto. Posteriormente se añadieron algunas preguntas para comprender mejor el afrontamiento en la esfera familiar.

Las entrevistas se realizaron por una enfermera con perfil investigador y con experiencia en dicha metodología (ETM). Estas se llevaron a cabo en una sala del hospital, fueron grabadas en audio y su duración estimada fue de una hora.

Los datos sociodemográficos se extrajeron de la base de datos del estudio previo registrado en la plataforma electrónica Clinapsis ${ }^{\odot}$.

\subsection{Análisis de los datos}

La investigadora principal (ETM) llevó a cabo el análisis y este se discutió con otra investigadora del equipo (NCB) para lograr posibles interpretaciones y categorías relevantes. Se realizó un análisis de contenido temático (Hsieh \& Shannon, 2005) asistido por el programa Nvivo 12 (v. 12). El uso de este programa informático aportó a nuestra investigación calidad, credibilidad y economía (Trigueros Cervantes, Rivera García, \& Rivera Trigueros, 2018).

En una primera fase del análisis se llevó a cabo la categorización teórica conceptual de las entrevistas. La categoría principal o Nodo principal (terminología Nvivo) se elaboró a partir de la teoría formal y la escala de Modos de Afrontamiento (Ways of Coping, WOC) (Lazarus \& Folkman, 1984). A partir de esta se identificaron en las entrevistas 8 estrategias diferentes de afrontamiento: 1) Confrontación, 2) Planificación, 3) Distanciamiento, 4) Autocontrol, 5) Aceptación de responsabilidad, 6) Escape-Evitación, 7) Reevaluación positiva, y 8) Búsqueda de apoyo social. Además de las estrategias se identificó la fase de la enfermedad en la que se utilizó cada estrategia: fase aguda (etapa del diagnóstico), fase extendida (intervención y tratamiento), y fase permanente (desde que se finaliza el tratamiento adyuvante, quimioterapia o radioterapia, hasta dos años después). Esta clasificación 
temporal se basó en la realizada por Mullan sobre las etapas de supervivencia del cáncer (Mullan, 1985).

En una segunda fase del análisis se inició la codificación teórica deductiva en base a la categorización previamente explicada. A cada categoría o nodo se le asignó una unidad de significado, un fragmento de la entrevista que representara la idea o concepto de la categoría. Este proceso no fue excluyente, por lo que había fragmentos que podían pertenecer a dos o más categorías de forma simultánea.

Además, paralelamente a la codificación, se continuaron identificando nuevas categorías (emergentes) de tipo descriptivo que permitieron identificar principalmente comportamientos (afrontamiento del entorno, no comunicar la enfermedad), relaciones interpersonales (apoyo social, fuentes de apoyo), y situaciones (consecuencias de la enfermedad). También encontramos alguna categoría categorial que llega a abarcar un nivel explicativo (miedo) (Gibbs, 2012).

A medida que avanzó la codificación, se fue configurando el árbol de categorías y el patrón de análisis. Una vez finalizado el proceso de codificación se llevó a cabo la exploración de los datos a través de matrices de codificación que permitieron generar y comprobar la relación entre las categorías principales: las estrategias de afrontamiento y las fases de la enfermedad. El resultado de la matriz mostró la cantidad de unidades de significado o fragmentos codificados dentro de las dos categorías simultáneamente (Matriz de codificación de intersección) y permitió explicar los cambios de las EA en las mujeres y su papel en la adaptación al cáncer de mama.

La validación de los resultados finales se realizará mediante la triangulación con una profesional experta en la materia como es la enfermera de la Unidad de Mama del hospital.

\section{RESULTADOS}

\subsection{Características de las participantes}

Durante los meses de octubre de 2018 y junio de 2019 se realizaron un total de trece entrevistas a mujeres diagnosticadas de cáncer de mama. A continuación, se muestran resultados preliminares que incluyen los discursos de mujeres con edades comprendidas entre los 50 y 59 años con diferentes ingresos familiares, y mujeres con edades entre los 60 y 70 años con ingresos familiares medios y altos. 
La media de edad de las mujeres en el momento del diagnóstico fue de 59 años y en el momento de la entrevista de 62 años. El 100\% fueron intervenidas de tumorectomía, y excepto una mujer todas recibieron radioterapia; además, el $46 \%$ también recibió quimioterapia. En cuanto a los ingresos familiares, el 15\% de las mujeres tenían ingresos bajos, el $46 \%$ ingresos medios y el $38 \%$ ingresos altos.

\subsection{Evolución de las estrategias de afrontamiento (EA) según la fase de la enfermedad.}

\subsubsection{Fase aguda: "Yo hacía mi vida normal, super estresada, que yo creo que de eso mismo me vino"}

En la fase aguda encontramos heterogeneidad en el uso de EA. La estrategia de aceptación de responsabilidad aparece exclusivamente en esta fase, mientras que la de reevaluación positiva es la única que no se identifica.

La estrategia de confrontación fue utilizada como rechazo al tratamiento ante diagnósticos de buen pronóstico. La estrategia de planificación también estuvo apoyada por el buen pronóstico de la enfermedad, el cual motivó actitudes positivas para afrontar el tratamiento. Por otra parte, encontramos la estrategia de distanciamiento, que se relacionó sobre todo con evitar hablar del tema con la familia para no preocupar. La estrategia de autocontrol también se apoyó en el pronóstico y en la atención sanitaria.

En esta fase encontramos la estrategia de aceptación de responsabilidad, ya que las mujeres asocian la enfermedad a un exceso de estrés, o al periodo de la jubilación. Por otro lado, de la estrategia de escape-evitación encontramos pocas referencias, aun así, una mujer refirió hundirse y necesitó tratamiento antidepresivo, y otra utilizó un pensamiento excesivamente positivo. Por último, la búsqueda de apoyo social fue la estrategia más utilizada durante el diagnóstico. Encontramos mujeres con amigas o conocidas que han sufrido la enfermedad y refieren su apoyo como un pilar clave en esta etapa:

“Llamé a la puerta, pregunté porque yo no sabía ni cómo se llamaba, y me presenté en su casa. (...) "no que me ha dicho Antonia que a ti también te han encontrado un tumor en el pecho" y me dice "a mí en dos", pero riéndose ella "a mí en los dos (...) sí pero eso no pasa nada, no pasa nada, ¿pero estás preocupada?" y le digo "pues yo estoy asustadísima" "que no mujer que eso nos lo van a quitar" ¿pero sabes? con una moral..." E.4.

\subsubsection{Fase Extendida: "Cuando vi que todo se ponía en marcha la confianza fue absoluta"}


En esta fase predominan estrategias de búsqueda de apoyo social, de distanciamiento y de planificación. Sin embargo, no encontramos las estrategias de aceptación de responsabilidad ni de reevaluación positiva.

La confrontación es una estrategia poco utilizada por las mujeres y continua en la misma línea que en la fase aguda. La estrategia de planificación se centró en abordar las consecuencias del tratamiento y en alguna ocasión, en la que la enfermedad convivió con una situación familiar delicada, la mujer se esforzó en organizar su actividad para atender a la otra persona. El cuidado a los demás se puede clasificar también como una estrategia de distanciamiento. Sin embargo, como distanciamiento en esta fase, predominan conductas para mantener la "normalidad":

“Las preocupaciones que tienes son... mi hija se iba un año a vivir a Estados Unidos porque acababa la carrera (...) entonces ella no se quería ir... Para mí era más importante que todo continuara con normalidad, (...) Y eso es lo que a veces me costó más." E.3

La estrategia de autocontrol las mujeres la utilizaban para asimilar la enfermedad, para controlar los sentimientos durante el tratamiento. La estrategia de escape-evitación se identifica en diferentes conductas: una de las mujeres refiere que durante el tratamiento salía mucho y compró de forma compulsiva, otra mujer refiere ir al tratamiento con una positividad que ahora considera excesiva, y por último un par de mujeres recurrieron a tratamiento antidepresivo. Por otra parte, al igual que en la fase aguda, una de las estrategias más utilizadas fue la búsqueda de apoyo social.

\subsubsection{Fase permanente: "Distanciarme, querer aparcar eso que ha sido una etapa de mi vida"}

En esta fase ocurre al contrario que en la fase aguda, la estrategia que únicamente encontramos en este periodo es la reevaluación positiva, sin embargo, no aparece la estrategia de aceptación de la responsabilidad. La estrategia que predomina en esta fase es la de distanciamiento, seguida de la búsqueda de apoyo social.

La estrategia de confrontación la encontramos en una ocasión y se utilizó para rechazar el tratamiento con hormonoterapia. La estrategia de planificación se relacionó sobre todo con la actitud de las mujeres ante las consecuencias a largo plazo de la enfermedad. Por otra parte, la estrategia que predomina en esta fase es la de distanciamiento. Las mujeres refieren evitar hablar de la enfermedad, e incluso manifiestan su voluntad de salir de grupos on-line sobre cáncer de mama: 
“(...) A veces pienso "me voy a ir" [del grupo de Facebook] pero no, no porque yo qué sé, tampoco quiero hacerles el feo a ellas ¿no? Y me consta que hay quién lo ha pasado peor que yo, pero 50 veces". E. 8

La estrategia de autocontrol las mujeres la utilizan para intentar aceptar la enfermedad, asumir las limitaciones y controlar el miedo a las recaídas. En relación a esto encontramos el uso de otra estrategia, la de escape-evitación. Por otra parte, una estrategia propia de la fase permanente es la estrategia de reevaluación positiva.

"Porque me quiero más, me quiero mucho, me quiero mucho. Y cualquier cosita ahora por poco que pueda la hago, antes no, yo era la Cenicienta de mi casa (...)". E.9

Por último, la estrategia de búsqueda de apoyo social se utiliza para dar respuesta a las limitaciones tras la enfermedad, o para hablar de la enfermedad y del miedo que comporta. Gran parte de las mujeres no encuentran satisfechas sus necesidades de apoyo en esta fase.

\section{DISCUSIÓN Y CONCLUSIONES}

Coincidiendo con estudios previos, los resultados preliminares muestran diversas EA en cada fase, y cada mujer recurre a unas u otras, e incluso a varias a la vez (Dunkel-schetter, Feinstein, Taylor, \& Falke, 1992; Font \& Cardoso, 2009; Moos \& Holahan, 2007; Roussi, Krikeli, Hatzidimitriou, \& Koutri, 2007). Aunque no existe un patrón común para todas las mujeres entrevistadas, sí que se aprecian coincidencias en el uso de unas EA frente a otras a lo largo del tiempo. Apoyándonos en Lazarus y Launier (1978) podemos llegar a comprender este fenómeno al entender que "la característica determinante de la respuesta a un estresor es la valoración individual de la situación" (Lazarus \& Launier, 1978, p. 302). Dicha reevaluación origina cambios en las demandas de la mujer que dan lugar a estrategias diferentes (Park \& Folkman, 1997).

En una primera fase inicial es donde se aprecia mayor variabilidad en el uso de las EA. Algunos estudios, acorde a nuestros resultados, han apreciado como en la fase de diagnóstico son diversas las EA utilizadas (Drageset et al., 2010; Roussi et al., 2007). Encontramos estudios que destacan reacciones de enfado y sentimiento de injusticia, y las mujeres atribuyen el diagnóstico a la voluntad de Dios (do Carmo Pinto Coelho Paiva et al., 2015; Hajian, Mehrabi, Simbar, \& Houshyari, 2017). Los resultados preliminares de este estudio difieren de esta afirmación y las mujeres atribuyen la enfermedad a un exceso de estrés o a un cambio laboral como la jubilación. Esto se reflejan en la estrategia aceptación 
de la responsabilidad, que únicamente se identifica en la fase aguda. Con ella las mujeres asumen cierto grado de control sobre la situación y llegan a pensar que si cambian ciertos hábitos y reducen el estrés pueden influir en su situación de salud (Moos \& Holahan, 2007).

En una segunda fase se identifican sobre todo estrategias de búsqueda de apoyo social, pero también de distanciamiento y planificación. La búsqueda de apoyo social se identifica como una estrategia que va ganando peso durante el diagnóstico y en el periodo del tratamiento llega a ser la estrategia más utilizada. El apoyo social recibido del entorno proporcionó a la mujer energía y motivación para adaptarse de forma exitosa a las diferentes etapas de la enfermedad (Williams \& Jeanetta, 2016).

En una última fase las mujeres atribuyen a la enfermedad diferentes significados como "dolor y angustia", "recurrencia", "supervivencia" y "consecuencias". La recurrencia se interpreta como una situación en la cual la mujer no puede intervenir, sino que tendría que aceptar. Este enfoque explicaría el uso de estrategias de distanciamiento en la fase permanente (Folkman, Lazarus, Dunkel-Schetter, DeLongis, \& Gruen, 1986). Sin embargo, aceptarse como superviviente, hace que la mujer valore el día a día e incluso aumente su autoestima y cuidado respecto a antes de la enfermedad. Esta actitud es propia de la estrategia de reevaluación positiva, presente únicamente en la fase permanente, y que se relaciona con bienestar psicológico de la mujer. (Drageset, Lindstrøm, \& Underlid, 2016; Kvillemo \& Bränström, 2014).

Como se puede apreciar, las EA varían y evolucionan pero no es posible determinar qué estrategia es más adaptativa según la fase de la enfermedad. Esto dependerá de la evaluación individual que cada mujer haga sobre la enfermedad, de las necesidades que se deriven de dicha evaluación y de las EA que satisfazcan dichas necesidades. A pesar de esto, los resultados preliminares han mostrado ciertas coincidencias en el uso de unas estrategias frente a otras y sobre ello valdría la pena profundizar en futuras investigaciones.

Abordar el estudio de las EA mediante investigación cualitativa ha permitido establecer una relación entre el significado que atribuyen las mujeres a su experiencia de vivir con cáncer de mama y el uso de EA en relación de dicha resignificación a lo largo del tiempo.

Conocer el uso de las EA en las mujeres diagnosticadas de cáncer de mama puede orientar a los profesionales de enfermería sobre las necesidades de dichas mujeres en cada fase de 
la enfermedad y permitir que se establezca una intervención específica en base al afrontamiento de cada mujer.

El presente trabajo muestra los resultados preliminares del estudio, por consiguiente, no se pueden establecer unas conclusiones concluyentes a la espera de finalizar el estudio.

Abreviaturas. ETM: Elena Torralba Martínez. NCB: Núria Codern Bové.

Agradecimientos. Los resultados preliminares presentados forman parte del estudio llevado a cabo por la Sra. Elena Torralba Martínez, la cual es candidata a doctorado en Metodología del Programa de Investigación Biomédica y Salud Pública, Universitat Autònoma de Barcelona, España. Agradecer la colaboración de Magda Ciendones, enfermera de la unidad de mama del hospital, en la selección de la muestra y como experta de referencia en la atención y cuidados a mujeres diagnosticadas de cáncer de mama.

\section{REFERENCIAS}

Bigatti, S. M., Steiner, J. L., \& Miller, K. D. (2012). Cognitive Appraisals , Coping and Depressive Symptoms in Breast Cancer Patients. Stress Health, 28, 355-361. https://doi.org/10.1002/smi.2444

Cha, K., Yoo, Y., \& Cho, O.-H. (2012). Stress and Coping Strategies of Breast Cancer Patients and their Spouses. Asian Oncol Nurs Vol., 12(1), 20-26.

Cope, D. G. (1995). Functions of a breast cancer support group as perceived by the participants: An ethnographic study. Cancer Nursing, 18(6), 472-478.

do Carmo Pinto Coelho Paiva, A., de Oliveira Salimena, A. M., de Oliveira Souza, Í. E., \& de Melo, M. C. S. C. (2015). Significado do diagnóstico de neoplasia mamária: Compreensão fenomenológica de mulheres. Revista Baiana de Enfermagem, 29(1), 59-67. Retrieved from http://search.ebscohost.com/login.aspx?direct=true\&db=fua\&AN=108763445\&lang=es\&site=ehost-live

Drageset, S., Lindstrøm, T. C., \& Underlid, K. (2010). Coping with breast cancer: Between diagnosis and surgery. Journal of Advanced Nursing, 66(1), 149-158. https://doi.org/10.1111/j.1365-2648.2009.05210.x

Drageset, S., Lindstrøm, T. C., \& Underlid, K. (2016). "I just have to move on": Women's coping experiences and reflections following their first year after primary breast cancer surgery. European Journal of Oncology Nursing, 21, 205-211. https://doi.org/10.1016/j.ejon.2015.10.005

Dunkel-schetter, C., Feinstein, L. G., Taylor, S. E., \& Falke, R. L. (1992). Patterns of Coping With Cancer. Health Psychology, 11(2), 79-87.

Edward, K., Chipman, M., Giandinoto, J., \& Robinson, K. (2019). Quality of life and personal resilience in the first two years after breast cancer diagnosis: systematic integrative review. British Journal of Nursing, 28(10).

Everly, G. S. (1989). The Anatomy and Physiology of the Human Stress Response. In A Clinical Guide to the Treatment of the Human Stress Response (pp. 17-51). New York, NY: Plenum Press. https://doi.org/10.1007/978-1-4613-0741-9

Ferrell, A. B. R., Dow, K. H., \& Grant, M. (1995). Measurement of the Quality of Life in Cancer Survivors. Springer, 4, 523-531.

Folkman, S., Lazarus, R. S., Dunkel-Schetter, C., DeLongis, A., \& Gruen, R. J. (1986). Dynamics of a Stressful Encounter. Cognitive Appraisal, Coping, and Encounter Outcomes. Journal of Personality and Social Psychology, 50(5), 992-1003. https://doi.org/10.1037/0022-3514.50.5.992

Font, A., \& Cardoso, A. (2009). AFRONTAMIENTO EN CÁNCER DE MAMA : PENSAMIENTOS , CONDUCTAS Y REACCIONES EMOCIONALES. Psicooncología, 6, 27-42. 
Gibbs, G. (2012). El anàlisis de datos en investigación cualitativa. Ediciones Morata.

GLOBOCAN. (2018). Estimated age-standardized incidence rates (World) in 2018, all cancers, both sexes, all ages. Retrieved from https://gco.iarc.fr/today/online-analysismap?v=2018\&mode=population \&mode_population=continents\&population=900\&populations $=900 \& k e y=a s r$ \&sex=0\&cancer $=39 \&$ type $=0 \&$ statistic $=5 \&$ prevalence $=0 \&$ population_group $=0 \&$ ages_group $\% 5 \mathrm{~B} \% 5 \mathrm{D}=0$ \&age S_group $\% 5 B \% 5 D=17 \& n b \_$items=5\&gro

Hajian, S., Mehrabi, E., Simbar, M., \& Houshyari, M. (2017). Coping Strategies and Experiences in Women with a Primary Breast Cancer Diagnosis. Asian Pacific Journal of Cancer Prevention, 18(1), $215-224$. https://doi.org/10.22034/APJCP.2017.18.1.215

Hsieh, H. F., \& Shannon, S. E. (2005). Three approaches to qualitative content analysis. Qualitative Health Research, 15(9), 1277-1288. https://doi.org/10.1177/1049732305276687

Jang, M., \& Kim, J. (2018). A structural model for stress, coping, and psychosocial adjustment: A multi-group analysis by stages of survivorship in Korean women with breast cancer. European Journal of Oncology Nursing, 33, 41-48. https://doi.org/10.1016/j.ejon.2018.01.004

Kaur, B., Stephen, J., Mackenzie, G., Doll, R., Cristina, M., \& Cadell, S. (2008). Understanding Canadian Punjabispeaking South Asian women's experience of breast cancer: A qualitative study. International Journal of Nursing Studies, 45, 266-276. https://doi.org/10.1016/j.ijnurstu.2006.08.023

Kvillemo, P., \& Bränström, R. (2014). Coping with breast cancer: A meta-analysis. PLoS ONE, 9(11), 1-26. https://doi.org/10.1371/journal.pone.0112733

Lazarus, R. S., \& Folkman, S. (1984). Stress , Appraisal, And Coping. New York, NY: Springer Publishing Company.

Lazarus, R. S., \& Launier, R. (1978). Stress-Related Transactions between Person and Environment. In Perspectives in Interactional Psychology (pp. 287-327). New York, NY: Plenum Press. https://doi.org/10.1007/978-1-4613-3997-7_12

Ley Orgánica 15/1999, de 13 de diciembre, de Protección de Datos de carácter personal, Boletín Oficial del Estado, 298 § 43088 (1999).

Moos, R. H., \& Holahan, C. J. (2007). Adaptative task and methods of coping with illnes and Disability. In E. Martz \& H. Livneh (Eds.), Coping with Chronic Illness and Disability (pp. 107-126). New York, NY: SpringerVerlag. https://doi.org/10.1007/978-1-4614-3399-6_5

Mullan, F. (1985). Seasons of survival: reflections of a physician with cancer. New England Journal of Medicine, 313, $270-273$.

Paek, M.-S., Ip, E., Levine, B., Avis, N., Ip, E. H., \& Avis, N. E. (2016). Longitudinal Reciprocal Relationships Between Quality of Life and Coping Strategies Among Women with Breast Cancer. Annals of Behavioral Medicine, 50(5), 775-783. https://doi.org/10.1007/s12160-016-9803-y

Palacios Espinosa, X., Lizarazo, A. M., Moreno, K. S., \& Ospino, J. D. (2015). El significado de la vida y de la muerte para mujeres con cáncer de mama. Avances En Psicología Latinoamericana, 33(3), 455-479. https://doi.org/10.12804/revistas.urosario.edu.co/apl/a.3221

Park, C. L., \& Folkman, S. (1997). Meaning in the context of stress and coping. Review of General Psychology, 1(2), 115-144. https://doi.org/10.1037/1089-2680.1.2.115

Roussi, P., Krikeli, V., Hatzidimitriou, C., \& Koutri, I. (2007). Patterns of coping, flexibility in coping and psychological distress in women diagnosed with breast cancer. Cognitive Therapy and Research, 31(1), 97-109. https://doi.org/10.1007/s10608-006-9110-1

Trigueros Cervantes, C., Rivera García, E., \& Rivera Trigueros, I. (2018). Técnicas conversacionales y narrativas Investigación cualitativa con Software NVivo. (E. andaluza de S. Pública, Ed.). Granada (España).

Williams, F., \& Jeanetta, S. C. (2016). Lived experiences of breast cancer survivors after diagnosis, treatment and beyond: Qualitative study. Health Expectations, 19(3), 631-642. https://doi.org/10.1111/hex.12372

World Medical Association. (2008). Declaration of Helsinki: ethical principles for medical research involving human subjects. https://doi.org/10.1093/acprof:oso/9780199241323.003.0025

Zhang, Q., Xiao, S., Yan, L., Sun, L., Wang, Y., \& Huang, M. (2019). Psychosocial Predictors of Adjustment to Disability Among Patients With Breast Cancer: A Cross-Sectional Descriptive Study. Journal of Nursing Research (Lippincott Williams \& Wilkins), 27(2), e15-e15. https://doi.org/10.1097/jnr.0000000000000283 